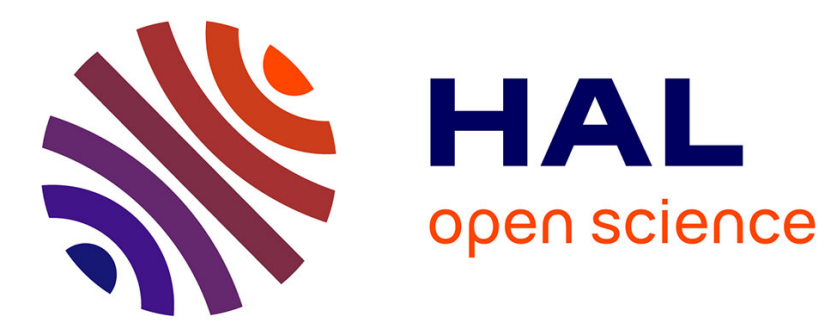

\title{
Film drainage of viscous liquid on top of bare bubble: Influence of the Bond number
}

Helena Kocarkova, Florence Rouyer, Franck Pigeonneau

\section{To cite this version:}

Helena Kocarkova, Florence Rouyer, Franck Pigeonneau. Film drainage of viscous liquid on top of bare bubble: Influence of the Bond number. Physics of Fluid, 2013, 25 (022105), pp.1. 10.1063/1.4792310 . hal-00795487

\section{HAL Id: hal-00795487 \\ https://hal-enpc.archives-ouvertes.fr/hal-00795487}

Submitted on 28 Feb 2013

HAL is a multi-disciplinary open access archive for the deposit and dissemination of scientific research documents, whether they are published or not. The documents may come from teaching and research institutions in France or abroad, or from public or private research centers.
L'archive ouverte pluridisciplinaire HAL, est destinée au dépôt et à la diffusion de documents scientifiques de niveau recherche, publiés ou non, émanant des établissements d'enseignement et de recherche français ou étrangers, des laboratoires publics ou privés. 


\section{AIP Fluids}

Film drainage of viscous liquid on top of bare bubble: Influence of the Bond number

Helena Kočárková, Florence Rouyer, and Franck Pigeonneau

Citation: Phys. Fluids 25, 022105 (2013); doi: 10.1063/1.4792310

View online: http://dx.doi.org/10.1063/1.4792310

View Table of Contents: http://pof.aip.org/resource/1/PHFLE6/v25/i2

Published by the American Institute of Physics.

\section{Related Articles}

The effect of viscoelasticity on the dynamics of gas bubbles near free surfaces

Phys. Fluids 25, 022104 (2013)

Enhancement of momentum coupling coefficient by cavity with toroidal bubble for underwater laser propulsion J. Appl. Phys. 113, 063107 (2013)

Frequency dependence and frequency control of microbubble streaming flows

Phys. Fluids 25, 022002 (2013)

Controlling bubble motion over heated surface through evaporation momentum force to enhance pool boiling heat transfer

Appl. Phys. Lett. 102, 051611 (2013)

Dynamics of micro-bubble sonication inside a phantom vessel

Appl. Phys. Lett. 102, 013702 (2013)

\section{Additional information on Phys. Fluids}

Journal Homepage: http://pof.aip.org/

Journal Information: http://pof.aip.org/about/about_the_journal

Top downloads: http://pof.aip.org/features/most_downloaded

Information for Authors: http://pof.aip.org/authors

\section{ADVERTISEMENT}

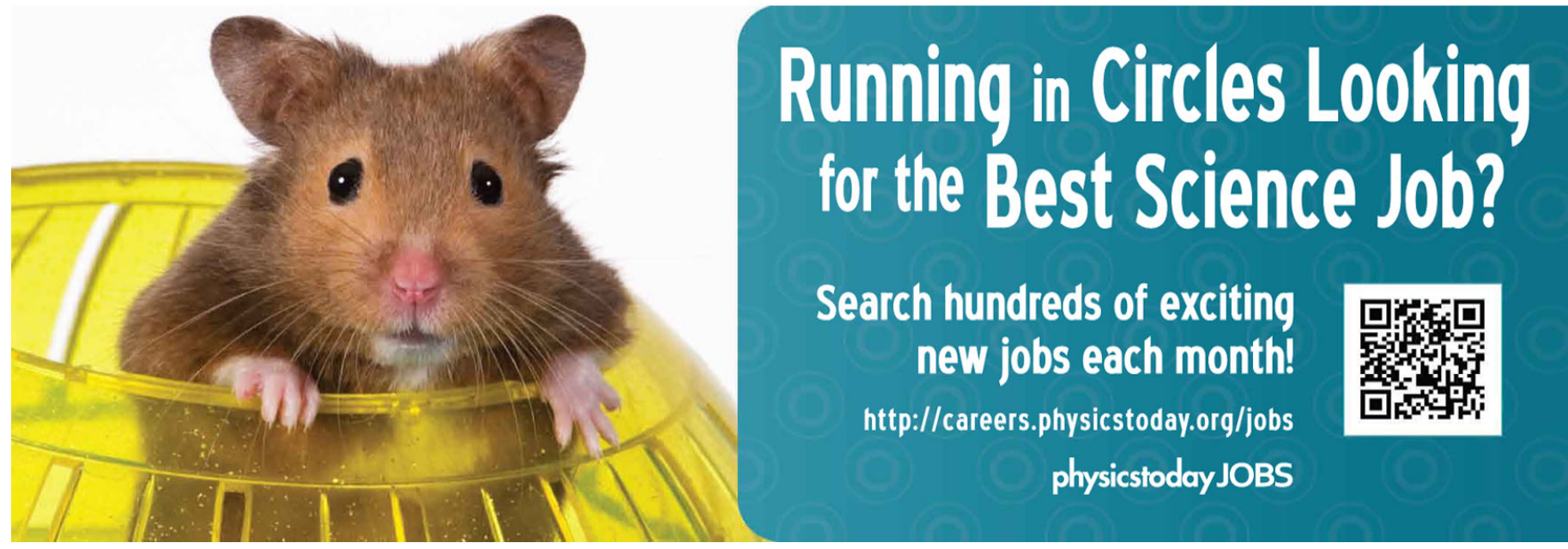




\title{
Film drainage of viscous liquid on top of bare bubble: Influence of the Bond number
}

\author{
Helena Kočárková, ${ }^{1}$ Florence Rouyer, ${ }^{2,3}$ and Franck Pigeonneau ${ }^{1}$ \\ ${ }^{1}$ Surface du Verre et Interfaces, UMR 125 CNRS/Saint-Gobain, 39 Quai Lucien Lefranc, \\ BP 135, 93303 Aubervilliers Cedex, France \\ ${ }^{2}$ Université Paris-Est, Laboratoire Navier (UMR CNRS 8205, Ecole des Ponts Paris Tech, \\ IFSTTAR), 5 bd Descartes, 77454 Marne-la-Vallée, France \\ ${ }^{3}$ Université Paris-Est, Laboratoire de Physique des Matériaux Divisés et des Interfaces, \\ EA7264, 77454 Marne-la-Vallée, France
}

(Received 27 March 2012; accepted 21 January 2013; published online 25 February 2013)

\begin{abstract}
We present experimental results of film drainage on top of gas bubbles pushed by gravity towards the free surface of highly viscous Newtonian liquid with a uniform interface tension. The temporal evolution of the thickness of the film between a single bubble and the air/liquid interface is investigated via interference method. Experiments under various physical conditions (range of viscosities and surface tension of the liquid, and bubble sizes) evidence the influence of the deformation of the thin film on the thinning rate and confirm the slow down of film drainage with Bond number as previously reported by numerical work of Pigeonneau and Sellier [Phys. Fluids 23, 092102 (2011)]. Considering the liquid flow in the cap squeezed by buoyancy force of the bubble, we provide an approximation of thinning rate as a function of Bond number that agrees with experimental and numerical data. Qualitatively, the smaller the area of the thin film compare to the surface of the bubble, the faster the drainage. (c) 2013 American Institute of Physics. [http://dx.doi.org/10.1063/1.4792310]
\end{abstract}

\section{INTRODUCTION}

Foam is a disperse system made of gas bubbles separated by liquid films. Its useful properties lead to a large range of applications in cosmetic, food, automotive, building materials, and so on. ${ }^{1,2}$ Nevertheless, foam can have a negative impact in various processes as for instance in glass melting. ${ }^{3,4}$ Indeed, foam is a thermal insulator that decreases the energetic efficiency of glass furnaces. The conditions leading to the foam layer at the top of a glass bath is still scarcely understood and it requires fundamental investigations. The stability of foams in high viscous fluid occurs also in lava flows ${ }^{5}$ affecting the eruption mechanisms. ${ }^{6}$

For such fluids that a priori do not contain any surfactant to stabilize thin films and for which liquid flow is slow, the lifetime of foams is strongly dependent on the liquid drainage through films and Plateau borders. The first step in characterizing the liquid drainage is to determine the properties of the liquid flow confined by liquid-air interfaces. Practically, this can be achieved at the mesoscale by studying the drainage of thin film as it is presented in the book of Mysels et al. ${ }^{7}$

The study of thin film drainage gives information on the behavior of the liquid-air interface. With soap films attached to wire frames, Mysels et al. ${ }^{7}$ pointed out that when the surfactant concentration is high, the film drainage is slow because interfaces behaving like immobile boundaries. By solving hydrodynamic equations with the no-slip conditions, Schwartz and Roy ${ }^{8}$ found out an algebraic decay of the film thickness as a function of time. In contrary, with a relatively small concentration of surfactant, the film boundaries are mobile leading to a fast drainage. The lubrication models give an exponential decay of the film thickness with time. ${ }^{8}$

Equivalent experiments have been done on droplet at a liquid-liquid interface ${ }^{9-11}$ or on bubble at a free interface. ${ }^{12,13}$ The mobility of the fluid interfaces has an influence on the dynamics of the film drainage similar to vertical films. In absence of surfactants, mobility of the interface is associated with 
the viscosity ratio of the dispersed and the continuous phases. Fully mobile interfaces correspond to the case of bubbles, whereas immobile interface corresponds to viscous drop. Yiantsios and Davis ${ }^{14}$ achieved a theoretical work to determine the motion of drop towards solid or deformable interfaces using the lubrication theory. They found that a pocket of fluid is trapped in the center of the film during its drainage (formation of a dimple), and that film thins according to a fractional powers of time. Nevertheless, this work is only valid for a small deformation of the interfaces (drop size smaller than capillary length) and for ratio of viscosities of the inner and outer liquids larger than unity or of the order of unity. In case of viscosity ratio of the order of unity and for drop diameter comparable to the capillary length, recent experiments evidenced asymmetry of the dimple that would speed up the drainage compare to symmetric model prediction. ${ }^{15}$ In the limit of bubble size larger than the capillary length with mobile interface (no surfactants), Debrégeas et al. ${ }^{12}$ evidenced the exponential decay of the film as a function of time and they shown that the film thinning rate is proportional to radius of curvature of the film. Similarly, experiments with capillary force balance shown that curved film with immobile interfaces drains twice as slow as the plane-parallel film. ${ }^{16}$ Recently, Pigeonneau and Sellier ${ }^{17}$ studied by solving Stokes equations using a boundary integral formulation the migration of bubble with mobile interface and the drainage of thin curved film on top of it. They pointed out the exponential decay of the thin liquid film. Moreover, they shown the strong influence of the bubble size on the thinning rate.

In spite of these contributions that evidence effect of film curvature and bubble size on drainage, the bubble size effect has not yet been observed experimentally over a large range of physical parameters. In fact, few attentions have been paid to the coalescence of bubbles at the top of a bath with mobile interfaces compared to the numerous studies of the coalescence between drops. ${ }^{18}$ However, it is noteworthy that this step is crucial for the formation of a froth (foam layer) depending on the ratio of the production rate and death rate of bubbles at the free surface. Thus, the present experimental work studies film drainage on top of a bubble at the free surface of a viscous liquid for different bubble diameters around the capillary length. In addition, we explore viscous and capillary forces by varying surface tension and viscosity over more than a decade in a regime where inertia is negligible (low Reynolds number and high Ohnesorge number) using oils (UCON ${ }^{\mathrm{TM}}$ oil and Castor oil) and molten glasses (silicate melts). Moreover, molten glass studies require a specific experimental set-up working at high temperature which is always a big challenge. Even so, such industrial material is known to behave has classical Newtonian liquid but its rheology inside a thin film of less than few microns as well as the rheology of its interface is still poorly characterized. ${ }^{4}$ Thus, the secondary purpose of this investigation is to find a "liquid" to model molten glass in experiments carried out at room temperature.

First, the two experimental set-ups that allow measurements of film drainage on top of a bubble, over a wide range of physical parameters are presented in Sec. II. In Sec. III, the experimental results are developed and discussed in comparison with previous experimental works and recent numerical simulations. We propose a new scaling for film thinning rate on top of a bubble that is based on gravity driven flow and extensional viscous dissipation and that takes into account the variation of the geometry of the cap with the size of the bubble. Finally, conclusions are drawn in Sec. IV.

\section{EXPERIMENTAL SET-UP AND DETERMINATION OF THE PHYSICAL PARAMETERS}

\section{A. Presentation of physical parameters}

When a bubble rises through a liquid towards a free surface, three steps emerge. The first is the rising of the bubble driven by the buoyancy forces where gradually the bubble velocity decreases with the distance between the bubble and the free surface. ${ }^{17}$ In the second step, corresponding to the drainage, a liquid film is created between the bubble interface and the free surface which thickness decreases with the time. The third step is the film rupture and it is outside of the scoop of the present work. In the following, the parameters which control the shape and the drainage are presented.

The shape of the bubble is mainly controlled by the relative importance of the buoyancy force given by $\rho g \pi D^{3} / 6$ against the surface tension proportional to $D \sigma$ where $\rho$ is the liquid density (the gas density is neglected), $D$ is the bubble diameter, $\sigma$ is the surface tension, and $g$ is the gravitational 
constant. The ratio of these two forces defines the Bond number given by

$$
\text { Bo }=\frac{\rho g D^{2}}{\sigma} .
$$

During the film drainage, the bubble shape does not change with time. Assuming the axisymmetry, the film thickness, $h$, is a function of time, $t$ and the polar angle $\theta$. The film thickness depends also on $D, \rho, g, \sigma$, and $\mu$. Dimensional analysis by taking $\rho, D$, and $\mu$ as parameters with independent dimensions ${ }^{19}$ gives that

$$
\frac{h}{D}=f(\bar{t}, \theta, \text { Bo, Oh }),
$$

where $\bar{t}$ is the dimensionless time given by

$$
\bar{t}=\frac{t}{\tau}=\frac{\operatorname{tg} D}{v}
$$

The quantity $v$ is the kinematic viscosity, $\mu / \rho$ and $\tau$ is the characteristic viscous time. The dimensionless group, Oh, is the Ohnesorge number which compares viscous forces to inertia and capillary forces and is defined by

$$
\mathrm{Oh}=\frac{\mu}{\sqrt{\rho \sigma D}}
$$

In the previous works of Howell ${ }^{20}$ and more recently of Pigeonneau and Sellier, ${ }^{17}$ the dependence on the Ohnesorge number does not appear in the formulation meaning that the film thickness is only a function of $\bar{t}, \theta$, and Bo. The independence of Oh is tested in the present work.

Note that the time scale, $v /(g D)$, found in the dimensional analysis can be established by a scaling analysis. Indeed, without surfactant on the interface or in other words with fully mobile interfaces, the shear stresses are free and so the stress tensor is diagonal. ${ }^{21}$ Consequently, the motion is a purely extensional flow: the velocity profile in the film is a plug flow. ${ }^{22}$ As it has been already established for vertical film, ${ }^{8}$ the balance of gravity and viscous forces over a length scale equal to the diameter of the bubble $D$ given by

$$
\mu \frac{U}{D} \sim \rho g D
$$

which defines the velocity scale

$$
U=\frac{g D^{2}}{v},
$$

and the time scale

$$
\tau=\frac{v}{g D}
$$

This scaling time is identical to the previous one introduced in the dimensional analysis.

\section{B. Experiment at room temperature with polymer liquid and mineral oil}

Experiments are conducted in a room under air conditioning that fixes the atmospheric temperature at $20^{\circ} \mathrm{C}$. Bubbles are observed from top and side at the center of the horizontal surface of the liquid that fills a cylindrical cell as it is illustrated in Figure 1(a). The nature of liquids is described below in Subsection II E. The cell is made of teflon where its inner diameter and its height are, respectively, 3.4 and $3.5 \mathrm{~cm}$. The top view is recorded by a AVT Marlin CCD video camera at a maximum frame rate of 60 images/s. The side view is recorded by a Photon video camera (Fastcam 1024PCI $100 \mathrm{~K}$ ) at a frame rate within 100 and 10000 images/s depending on the velocity of the drainage. A powerful lighting (Dedolight 400D) is used in order to ensure a high rate recording with a low shutter time. Unfortunately, the device heats slightly the fluid up to $23 \pm 1.4^{\circ} \mathrm{C}$. Note that temperature measured at the liquid surface is uniform and it is controlled for each experiment. 

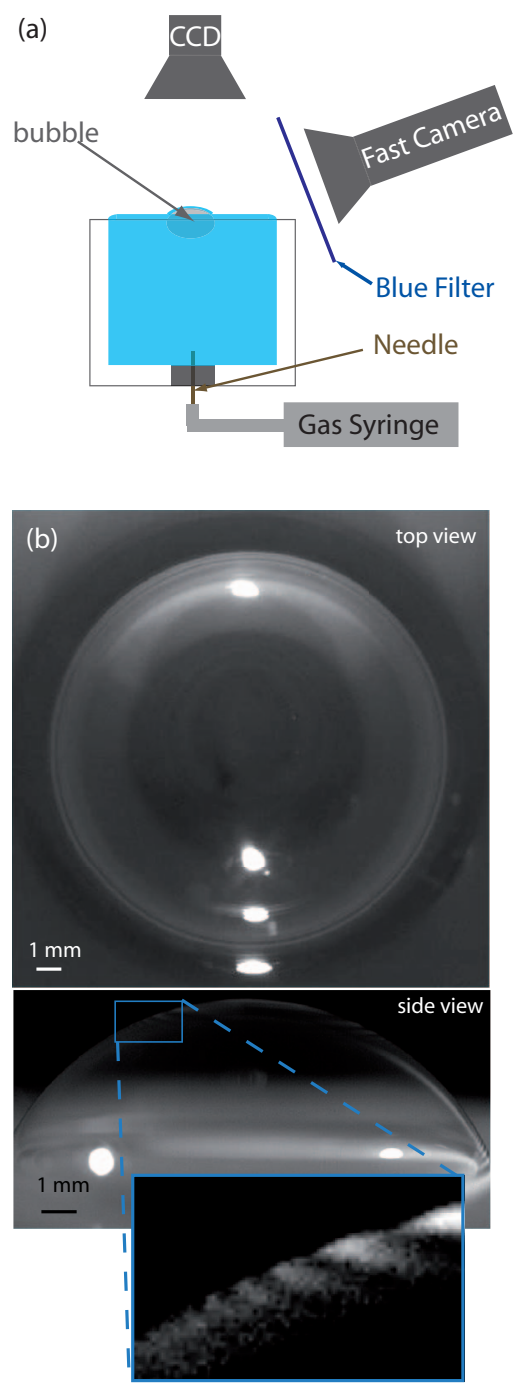

FIG. 1. (a) Sketch of the experimental set-up at room temperature. (b) Example of top and side views of a bubble at room temperature in $\mathrm{UCON}^{\mathrm{TM}}$ oil.

The bubbles are created by hand pushing air with a syringe through a needle placed vertically at the bottom of the cell. The bubble diameters range from 1 to $10 \mathrm{~mm}$ (cf. Table III) by varying the inner diameter of the needle from 0.4 to $1 \mathrm{~mm}$.

The bubble diameter is determined from top view images recorded during its ascension towards the free surface of the liquid before it deforms the interface. Measurement uncertainty is less than $4 \%$, see Figure 1(b).

Powerful white light illuminates from top side the surface of the liquid and many colored interference fringes are visible by eyes in the film on top of the bubbles. However, only the blue fringes are selected and recorded thanks to a blue filter placed in the front of the fast camera (see Figure 1(b)).

\section{Experiment at high temperature with molten glass}

In order to perform a similar experiment on molten glasses, a laboratory furnace has been designed. A sketch is given in Figure 2(a). This furnace is closed and composed by a circular alumina tube filled of air atmosphere. The heating is done with electrodes around the alumina tube. 
(a)

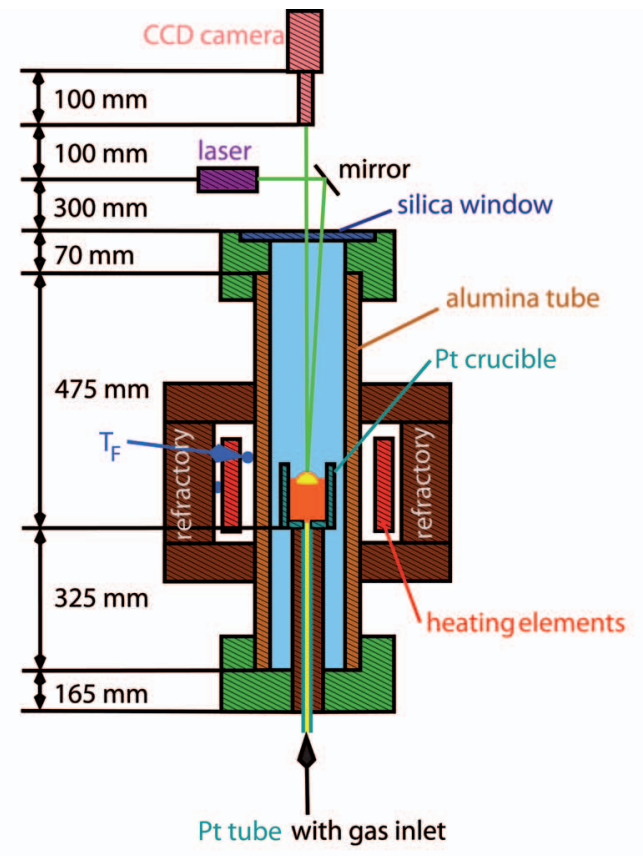

(b)

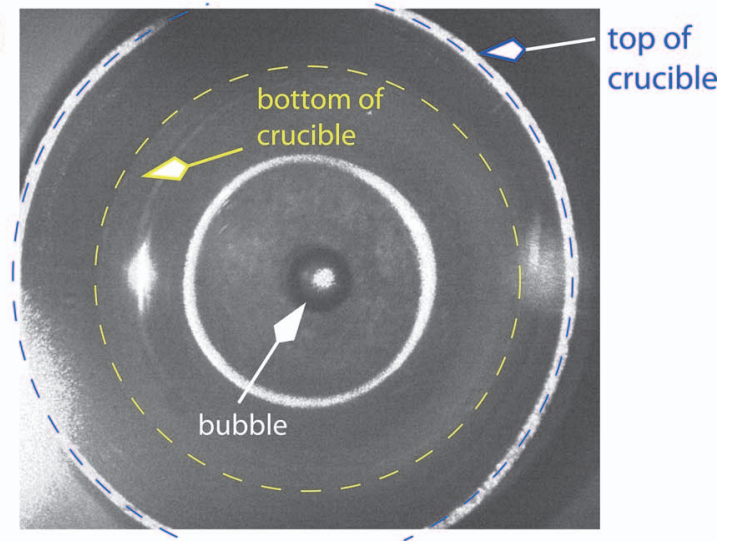

FIG. 2. (a) Sketch of the experimental set-up designed for molten glass in a furnace. (b) Example of top view of the platinum crucible with bubble $(\mathrm{D}=6.8 \mathrm{~mm})$ at $1044^{\circ} \mathrm{C}$ in molten window glass.

The temperature is recorded thanks to a thermocouple in the heating space, noted $T_{F}$ in Figure 2(a). The molten glass is introduced in the platinum crucible which is placed at middle height of the furnace. The real temperature at this position is lower than the temperature given by $T_{F}$ and has been calibrated previously, it is approximatively $65^{\circ} \mathrm{C}$ lower. Moreover, the thermal homogeneity has been verified showing that the thermal difference over the height of the crucible is not in excess of $5{ }^{\circ} \mathrm{C}$. The diameter and height of the crucible are 50 and $80 \mathrm{~mm}$, respectively, but glass fills less than a half of the crucible ( 30 or $40 \mathrm{~mm}$ ) to avoid the leakage of the melt in the furnace when creating bubbles. Gas is brought from $\mathrm{N}_{2}$ bottle through plastic (outside of the furnace) and platinum tube (inside the furnace) to a small hole $(100 \mu \mathrm{m})$ at the bottom of the crucible. The bubble inflation is controlled via a solenoid-valve that imposes periodically a pulse of pressure over an imposed time interval. The furnace allows visualization of the bubble only from the top through a silica window, see Figure 2(b).

The optical set-up, localized above the furnace, consists of laser, silver mirror, and CCD camera. Molten glass as well as refractories emit a thermal radiation in the infrared range of wavelength. Therefore, a green laser with wavelength at $532 \mathrm{~nm}$ is used in the experiment. The power of this laser is set to its maximum value at $150 \mathrm{~mW}$. In the front of the video camera, a narrow filter 
(a)
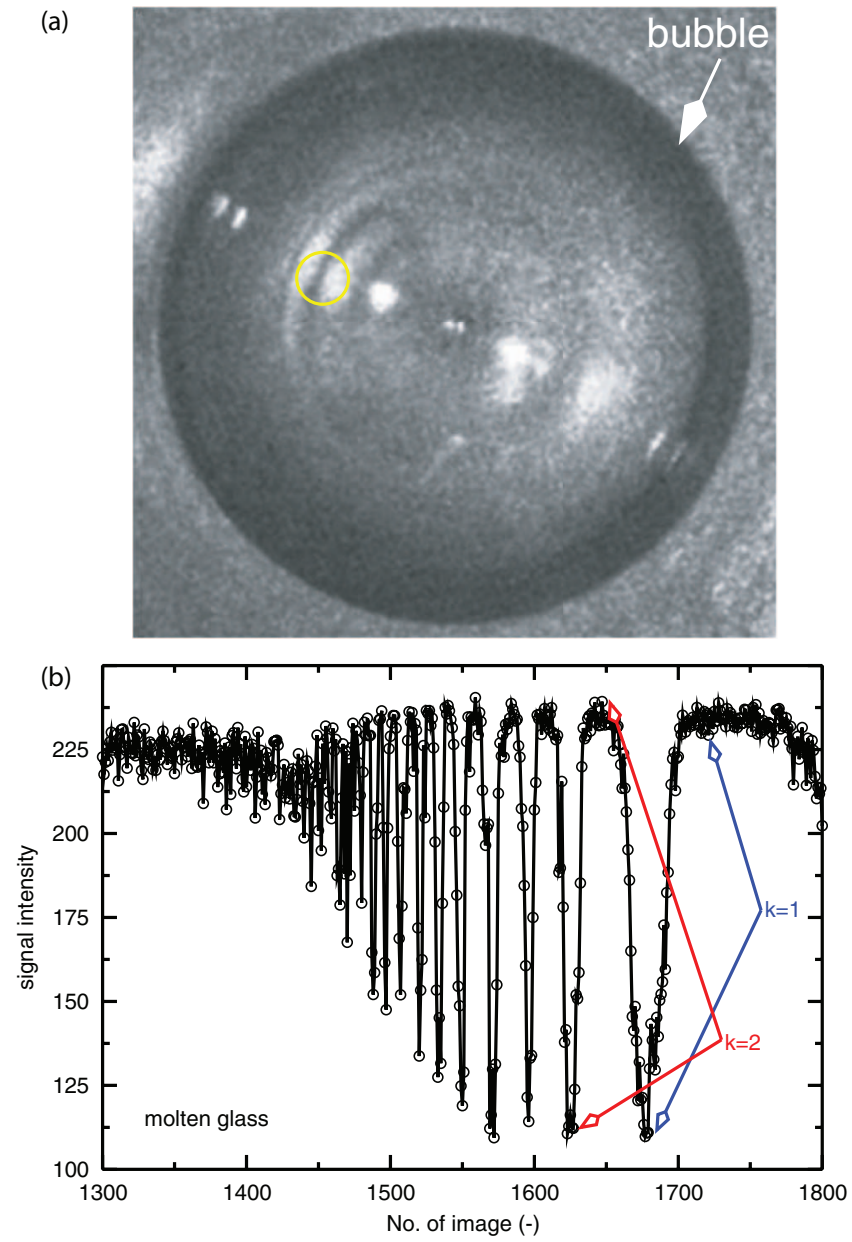

FIG. 3. (a) Example of an interference pattern on the bubble, signal in (b) was obtained as an average intensity on time in circled area. (b) Example of an interference signal of molten glass.

$\pm 1 \mathrm{~nm}$ centered in the wavelength of $532 \mathrm{~nm}$ is introduced. Moreover, in order to remove the specular reflexion of light, the crucible has been scratched. The resolution of the CCD camera was $1392 \times 1040$ pixel $^{2}$ with a maximal rate of 20 full frames per second.

\section{Interference analyses}

The evolution of thickness is computed from an interference signal, which is obtained from the images recorded during the experiment, see Figure 3. The maximum and the minimum of the light intensity according to the classical theory of light interference ${ }^{23}$ allows the determination of the film thickness as follows:

$$
\begin{gathered}
h_{I_{\max }}=\frac{\lambda(2 k-1)}{4 n}, \\
h_{I_{\min }}=\frac{\lambda k}{2 n},
\end{gathered}
$$

where $\lambda$ is the wavelength of the laser, $n$ is the refractive index, and $k$ is the order of the interference. Note that these equations are obtained assuming that the incident light is perpendicular to the interface, which is approximatively the case close to the apex of the bubble. 
TABLE I. Dynamic viscosity, density, surface tension, and refractive index of liquids explored in the experiments. The two first rows give the properties for liquids available at room temperature. The two last rows provide the properties for molten glasses determined for an operating temperature in the furnace equal to $1227^{\circ} \mathrm{C}$.

\begin{tabular}{lcccc}
\hline \hline & $\begin{array}{c}\mu \\
(\mathrm{Pa} \mathrm{s})\end{array}$ & $\begin{array}{c}\rho \\
\left(\mathrm{kg} \mathrm{m}^{-3}\right)\end{array}$ & $\begin{array}{c}\gamma \\
\left(\mathrm{m} \mathrm{m}^{-1}\right)\end{array}$ & $\begin{array}{c}n \\
(-)\end{array}$ \\
\hline Castor oil & $0.75 \pm 0.05$ & 957 & 33.8 & 1.46 \\
UCON $^{\mathrm{TM}}$ oil & $42 \pm 5$ & 1095 & 40.8 & 1.46 \\
Glass 1 & $64 \pm 7$ & 2344 & 322 & 1.52 \\
Glass 2 & $154 \pm 13$ & 2318 & 303 & 1.5 \\
\hline \hline
\end{tabular}

\section{E. Liquid properties and physical parameters}

For the experiments achieved at room temperature, the liquids are either polymer fluid, $\mathrm{UCON}^{\mathrm{TM}}$ 90 000, kindly provided by Dow Chemical or Castor oil purchased to Fischer Scientific. Physical properties of liquids are summed-up in Table I where the dynamic viscosity, the density, the surface tension, and the refractive index are provided. The main difference between these two oils is the dynamic viscosity since the $\mathrm{UCON}^{\mathrm{TM}}$ oil has a dynamic viscosity 56 times greater than the Castor oil.

Surface tension of oils are measured in the lab with pendant drop method (TECLIS). Dynamic viscosities of oils are measured by bubble ascension inside the crucible as well as with cone plate rheometer over the temperature range $21-25^{\circ} \mathrm{C}$. Error bars are related to temperature fluctuations from one experiment to another. Densities and refractive indexes of oil are taken from commercial data sheets provided by the suppliers.

For the experiments in the laboratory furnace at high temperature, two glasses are used. The compositions in main oxides are given in Table II. The Glass 1 is an oxide glass mainly composed of silica, soda ash, and lime. The Glass 2 is also an oxide glass for which $\mathrm{CaO}$ has been substituted by $\mathrm{K}_{2} \mathrm{O}$. Its quantity of alumina is also more important. The properties of these two glasses given in Table I has been determined for an operating temperature equal to $1227^{\circ} \mathrm{C}$ in the furnace. The relative large content in alumina leads to an increase of the dynamic viscosity of Glass 2 . The glass densities have been determined experimentally. Dynamic viscosities are determined from the glass composition according to the model of Lakatos et al. ${ }^{24}$ Surface tensions of glasses are also determined from their composition according to the computations provided by Dietzel, ${ }^{25}$ Lyon, ${ }^{26}$ Rubenstein, ${ }^{27}$ and Kucuk et al. ${ }^{28}$ The effect of the temperature on surface tension is estimated from the correction given by Scholze. ${ }^{29}$

The ranges of bubble diameter investigated during experiments are given in Table III. Larger viscosity and surface tension, larger the bubble size. We sum-up in Table III the Bond and Ohnesorge numbers. The range of Bond number does not change significantly from one liquid to another. Conversely, the range of Ohnesorge number is more important due to the large spread of dynamic viscosity.

\section{RESULTS AND DISCUSSION}

\section{A. Film drainage and influence of physical parameters}

Once the shape of the bubble is stable at the surface of the bath, the motion of circular interference fringes is observed from the center to the periphery of the bubble. Fringes are scarcely centered at the apex of the bubble, i.e., the center of the circular fringes do not perfectly coincide with the apex

TABLE II. Glass compositions given in weight percentage.

\begin{tabular}{lcccccc}
\hline \hline & $\mathrm{SiO}_{2}$ & $\mathrm{Na}_{2} \mathrm{O}$ & $\mathrm{CaO}$ & $\mathrm{K}_{2} \mathrm{O}$ & $\mathrm{MgO}$ & $\mathrm{Al}_{2} \mathrm{O}_{3}$ \\
\hline Glass 1 & 72.3 & 13.5 & 9.6 & $\ldots$ & 4 & 0.6 \\
Glass 2 & 61.8 & 12.6 & 0.5 & 9.4 & 7.6 & 8.1 \\
\hline \hline
\end{tabular}


TABLE III. Ranges of bubble diameter investigated in the experiments. The corresponding Bond and Ohnesorge numbers are also summarized.

\begin{tabular}{lccc}
\hline \hline & $D(\mathrm{~mm})$ & Bo & Oh \\
\hline Castor oil & $1.5-9.8$ & $0.8-27$ & $1.3-3.4$ \\
UCON $^{\mathrm{TM}}$ oil & $1.5-10.5$ & $0.6-32$ & $61.3-162$ \\
Glass 1 & $3-23$ & $6-32$ & $15-23$ \\
Glass 2 & $8-23$ & $5-40$ & $38-64$ \\
\hline \hline
\end{tabular}

of the bubble. These small decentering can be affected to a small misalignment of the optical set up compare to the vertical direction as well as a small asymmetric drainage. In this work, only results of circular fringes that encircled the top of the bubble are reported which corresponds to about two third of the experiments. A "black" film (no interference fringe) is observed all over the bubble just before its collapse. Thus, the last film thickness measured is estimated of the order of $200 \mathrm{~nm}$, note that due to uncertainties of the angle of incidence of the light we cannot give absolute values. The film thickness at its rupture is lower than this value.

From the interferometry, the film thickness is determined and plotted as a function of $\bar{t}$ defined by Eq. (3) in Figure 4 for (a) Castor oil, (b) $\mathrm{UCON}^{\mathrm{TM}}$ oil, (c) Glass 1, and (d) Glass 2. For all liquids, three experiments with three different bubble diameters are provided and for which the corresponding Bond numbers are reported in Figure 4. The film thickness is normalized by its maximal measured value corresponding to the interference fringe observed at the first time.

For all liquids, the film thickness decays exponentially with time meaning that the dimensionless thickness, $\bar{h}=h(\bar{t}) / h(0)$, can be written as follows:

$$
\bar{h}(\bar{t})=\exp (-a \bar{t}),
$$

where the coefficient $a$ can be seen as the logarithmic temporal derivative of $\bar{h}$ and thus the normalized thinning rate of the film. As previously pointed out by Schwartz and Roy ${ }^{8}$ and Debrégeas et al., ${ }^{12}$
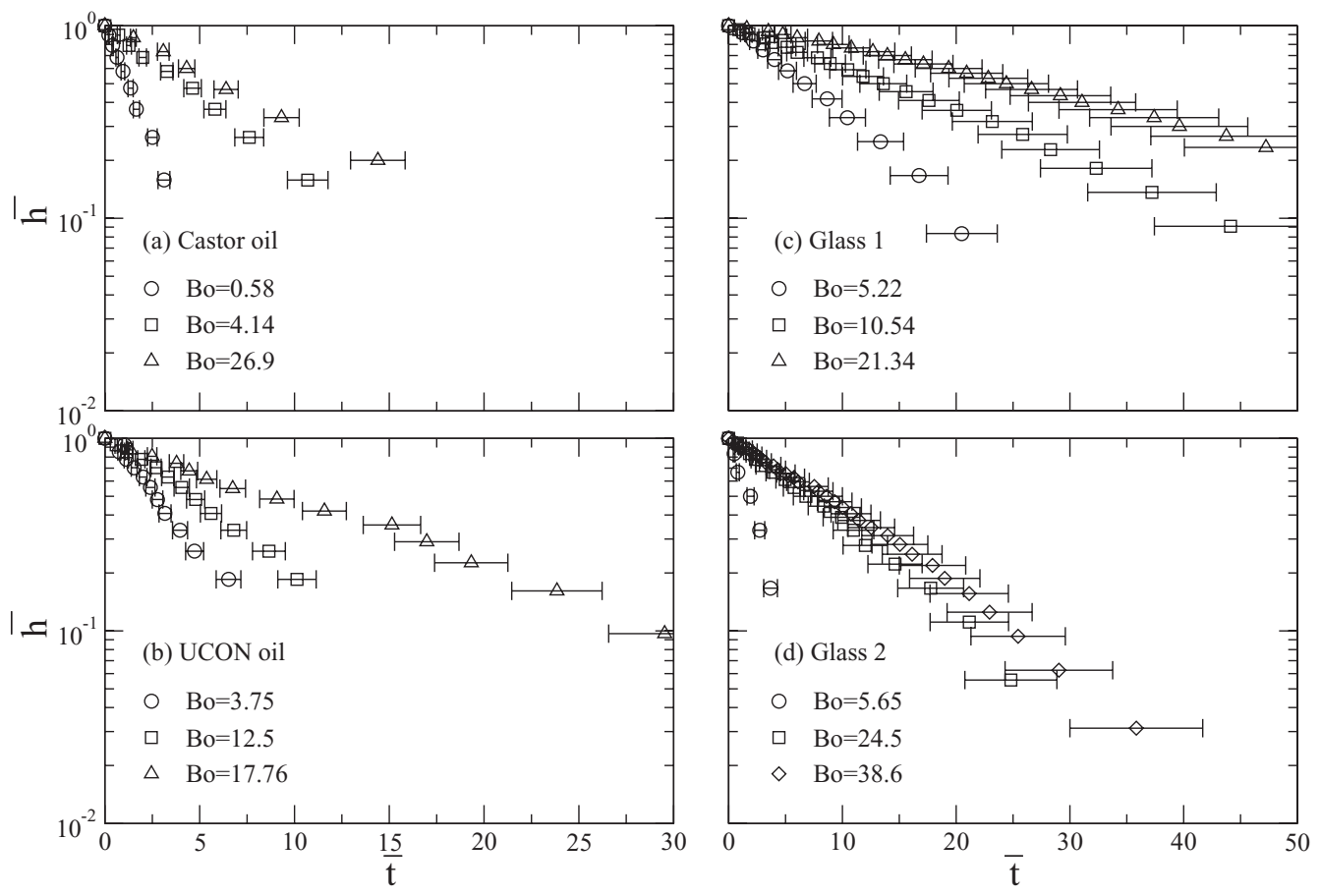

FIG. 4. Typical variation of the normalized thickness of the film as a function of dimensionless time. 

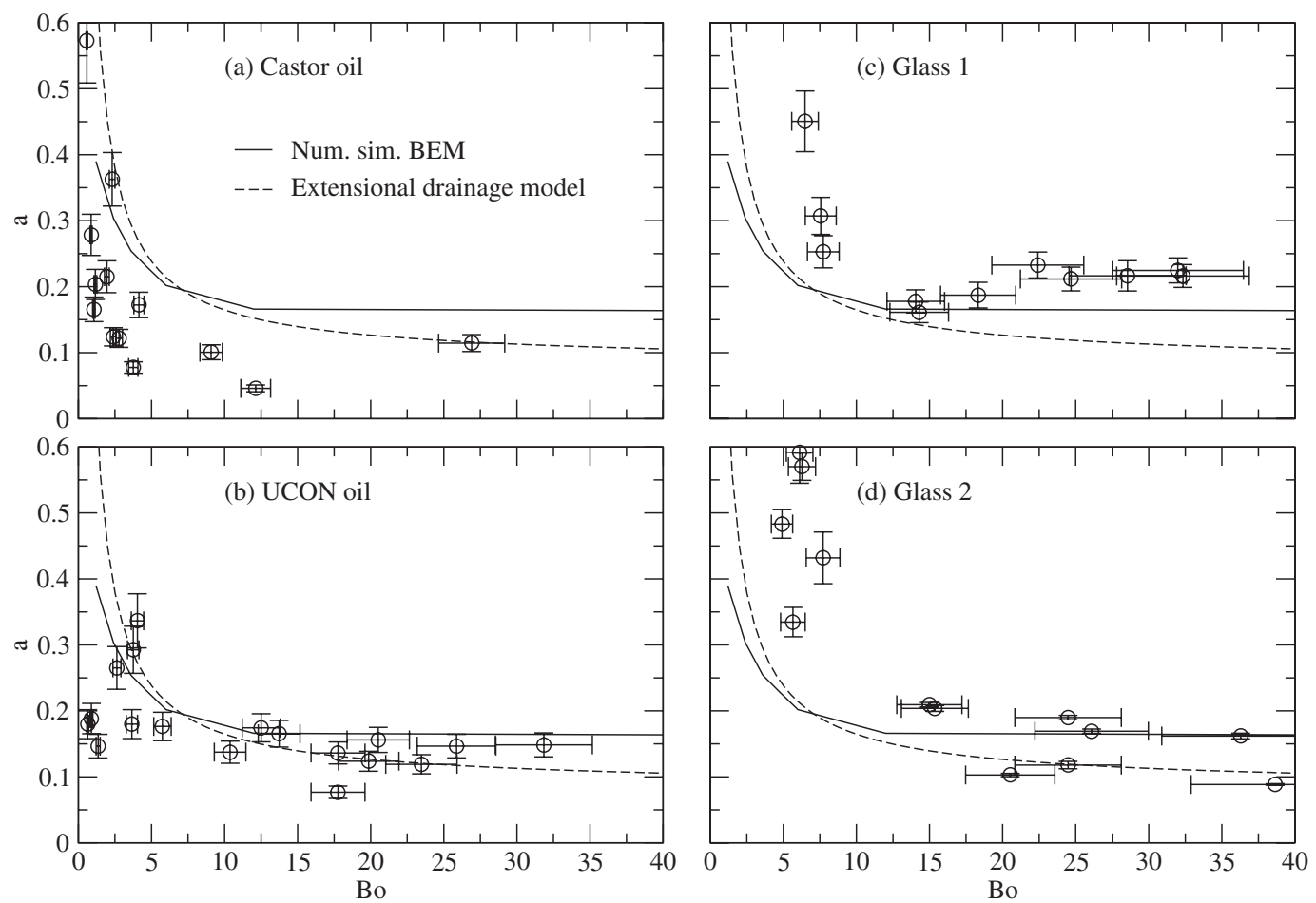

FIG. 5. Experimental results of normalized thinning rate versus Bond number obtaining for the four liquids.

the exponential behavior is the consequence of fully mobile interfaces. The liquid flow in the film is a purely extensional. These results then allow to conclude that none surface active agents affect the fluid interface for all liquids. Besides, normalized curves does not superimpose. Indeed, it appears from this graph that larger the bubble diameter, slower the decay of the thickness, i.e., lower $a$.

The thinning rate is determined from the experimental data using a regression fit assuming the exponential behavior. We thus plot the thinning rate, $a$, as a function of Bo for each liquid in Figure 5. Despite the scatter of the points, the trend of the decay of $a$ with Bo is clearly identified for small Bond number $(\mathrm{Bo} \leq 10)$ whatever the liquid nature. When the Bond number is larger than 10 , the thinning rate reaches an asymptotic value around 0.1 for all liquids.

Errors bars of $a$ due to uncertainties of physical parameters (viscosities and bubble diameters) do not seem to be enough to justify the spread of the data. Thus, in reference to a simple dimensional analysis (see Subsection II A), effects of viscous forces could be suspected as well. In aim to test this effect, we present in Table IV the value of $a$ and the Ohnesorge number estimated for bubbles for which the Bond number is close to 20 for the four liquids. No trend of variation of $a$ as a function of $\mathrm{Oh}$ is observed as considered by previous theoretical and numerical works. ${ }^{17,20}$

The scatter observed for the two glasses can be due to the underestimated uncertainties of the physical parameters for molten glass given by the literature. Moreover, the experiment at high temperature is not easy to do. The thermal field can perturb the drainage since the thermal gradient

TABLE IV. Values of $a$ and the corresponding Oh for the four liquids estimated Bo $\approx 20$.

\begin{tabular}{lcc}
\hline \hline & $a$ & Oh \\
\hline Castor oil & $0.1 \pm 0.05$ & $1.53 \pm 0.03$ \\
UCON $^{\mathrm{TM}}$ & $0.14 \pm 0.04$ & $65.7 \pm 1.3$ \\
Glass 1 & $0.2 \pm 0.05$ & $18 \pm 0.4$ \\
Glass 2 & $0.15 \pm 0.02$ & $44 \pm 6$ \\
\hline \hline
\end{tabular}


over the crucible which is relatively important, more than $500 \mathrm{~K}$ over few decimeters, can create a natural convection in the atmosphere. Similarly for experiments at room temperature, some air flow perturbation might occur above the bubble despite all precautions. Consequently, the drainage is not in a perfect axisymmetric and reproducible condition which can explain the observed scatter.

\section{B. Comparison with previous works}

An equivalent experiment has been previously done by Debrégeas $e t$ al. ${ }^{12}$ in silicone oil at very high viscosity, $10^{3} \mathrm{~Pa}$ s. Debrégeas et al. ${ }^{12}$ pointed out that the characteristic time from the bubble drainage depends on the radius of curvature of the cap. Their experimental work obtained for large bubble (limit of large Bond for which $R_{\text {cap }} \gg \kappa^{-1}$ ) shows that their thinning rate, $1 / \tau_{\text {drainage }}$ $=1.63 R_{\text {cap }}\left(\mathrm{s}^{-1} \mathrm{~m}^{-1}\right)$ which corresponds to a thinning rate $a=\tau / \tau_{\text {drainage }}$ equal to 0.104 . Their experimental results are in good agreement with our experimental results for Bo larger than 10. However, their model based on gravity-driven plug flow in a hemispherical cap of radius $R_{\text {cap }}$ predicts $1 / \tau_{\text {drainage }}=\left(\rho g R_{\text {cap }}\right) / \mu$ that corresponds to a variation of the thinning rate $a=R_{\text {cap }} / D$ from 1 to 0.63 for Bond number varying from zero to infinite (see the Appendix). This analytical model qualitatively agrees with our experimental data as it predicts a decrease of the thinning rate with Bo. However, the decay is less important than the experimental one (lesser than a factor of two all over the range of Bo compare to a factor larger than 4 for the experiments). Moreover, the analytical value of $a$ overestimates by a factor of 6 the experimental one at large Bond number. Thus, this "gravity-driven plug flow in hemispherical cap" model is not satisfactory to predict quantitatively experimental results.

Howell ${ }^{20}$ studied the drainage of bubble at the free surface using a lubrication model determining first the bubble shape according to the same principles of Princen. ${ }^{30}$ The behavior of the film thickness is established in the limit of small Bond number. Howell ${ }^{20}$ points out that the film thickness decays according to an algebraic function of time. Our experimental results show that we do not expect this trend. This disagreement suggests that the situation studied by Howell ${ }^{20}$ does not correspond to the real observation done experimentally.

On the other hand, our experimental results confirm the recent numerical results obtained by Pigeonneau and Sellier ${ }^{17}$ that are based on the boundary-integral formulation of Stokes equations and that point out an exponential behavior of the film thickness as a function of time. Quantitatively, the normalized thinning rate $a$ is deduced from an exponential fit to the numerical values of $\bar{h}(\bar{t})$ at long time and presented in Figure 5 by solid line. The decay of $a$ with Bo is clearly observed by the numerical method even so the thinning rate is underestimated for small Bond number due to a limitation of numerical method to compute accurately the drainage for a small Bond number. ${ }^{17}$ For a large Bond number, the numerical simulations show that the thinning rate is independent of Bo.

Finally, we recall that previous experiments done with polydimethylsiloxane (PDMS) did not give the same behaviors ${ }^{31}$ as the thinning rate was insensitive to the Bond number for the polymeric liquids. Different behavior might be artifacts due to the size of the polymeric chains but its understanding is still a challenge.

\section{Thinning rate approximation on top of a bubble and its dependance with Bond number}

Even if our experimental results agree with numerical simulations we do not have a simple relationship to determine the thinning rate. Therefore, in this subsection, a simple model to find an estimation of the thinning rate $a$ is proposed. We first assume that the thinning of the thin film on top of the bubble is due to the pressure imposed by the rising bubble and by the top interface that resists further deformation, once the fluid interfaces remain in stationary shape. However, the buoyancy force pulls the bubble upward and the capillary force pushes the interface downward, consequently the fluid contained in the film is squeezed. For film thickness much smaller than the radius of curvature of the bubble and assuming axisymmetry along the vertical axis, the tangential and the azimuthal stress can be neglected compare to the radial stress and thus the flow in the film is similar to the squeeze between two disks of area equal to $S_{\text {cap }}$. Assuming that the interfaces are 


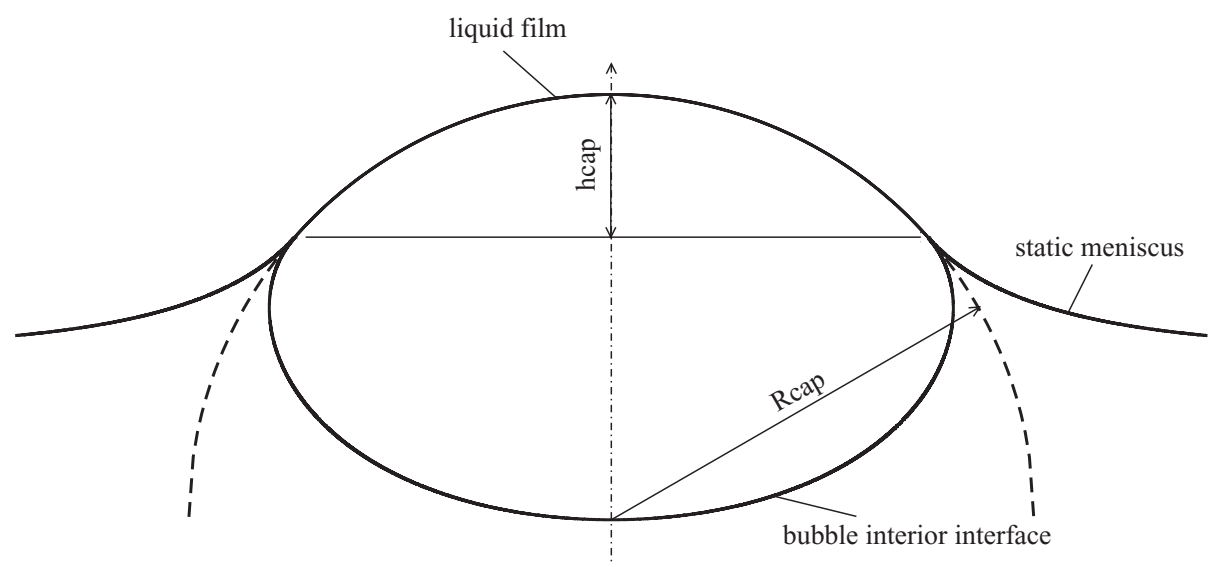

FIG. 6. Bubble shape at a free surface for $\mathrm{Bo}=1$.

parallel and fully mobile, the flow is a radial extension for which the tensile stress $\sigma_{T}$ can be written as follows:

$$
\sigma_{T}=6 \mu \dot{\epsilon},
$$

where the rate of strain, $\dot{\epsilon}$, is given by

$$
\dot{\epsilon}=\frac{-1}{2 h} \frac{d h}{d t} .
$$

This tensile stress is thus opposed to the pressure imposed by the buoyancy force of the bubble that apply to the cap

$$
\sigma_{T}=-3 \mu \frac{1}{h} \frac{d h}{d t}=+\frac{\rho g \frac{4 \pi}{3}(D / 2)^{3}}{S_{\text {cap }}}
$$

from which one can then deduce that the normalized thinning rate is

$$
a=\frac{2 \pi}{9} \frac{(D / 2)^{2}}{S_{\text {cap }}} .
$$

This equation involve the ratio of the surface of the cap $S_{\text {cap }}$ with the initial surface of spherical bubble that scales with $D^{2}$. The careful determination of the bubble shape at a liquid surface has been first described by Princen. ${ }^{30}$ His model decomposes interfaces in three distinguishable parts: (i) the interface between the interior of the bubble and the liquid bulk, (ii) the liquid film, and (iii) the static meniscus of the free surface of the liquid. As it was pointed out by Princen, the liquid film in the limit of very thin layer forms a spherical cap. Even if the problem is easy to formulate the practical determination is not so easy. The bubble shape for the Bond number equal to 1 is shown in Figure 6 in which the three distinguishable parts are pointed out. The geometry of the spherical cap is characterized by the radius of curvature, $R_{\text {cap }}$, the height of the cap, $h_{\text {cap }}$ and its area writes $S_{\text {cap }}$ $=2 \pi R_{\text {cap }} h_{\text {cap }}$. According to Princen's model, we determine the area of the spherical cap for Bond number varying from 0.05 to 200, and we plot $S_{\text {cap }}$ normalized by the square of bubble radius versus Bo in Figure 7. The normalized surface area of the cap increases with Bo. At small Bo, the interface above the bubble is slightly deformed and the bubble keeps the spherical shape and the surface of the cap tends to zero. Conversely, at large Bo, the free surface is strongly deformed and the shape of bubble as well, the surface of the cap tends to a hemispherical cap. We detailed in the Appendix this two asymptotic behaviors and give their analytical expression. In order to evaluate easily $S_{\text {cap }}$ which is useful in Eq. (14), we fit the curve using rational function for which the behaviors for small and 


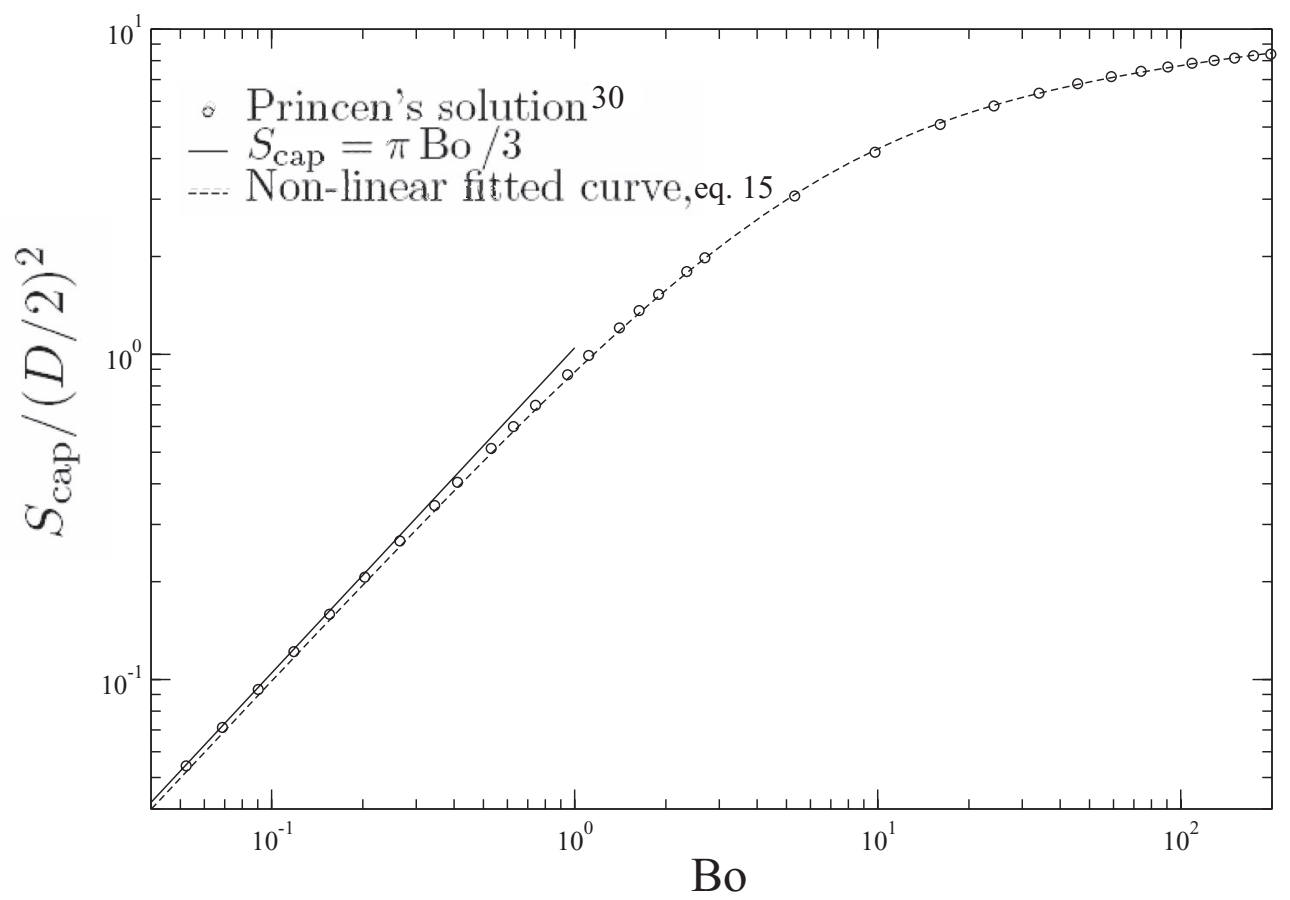

FIG. 7. $S_{\text {cap }} /(D / 2)^{2}$ versus Bo: circle points are determined according to Princen's model ${ }^{30}$ and dashed line is the fit corresponding to Eq. (15). The solid line is the asymptotic behavior at small Bond number according to Howell's model. ${ }^{20}$

large Bo are forced. This formula is given by

$$
\frac{S_{\text {cap }}}{(D / 2)^{2}}=2^{5 / 3} \pi \frac{94.7 \mathrm{Bo}+\mathrm{Bo}^{2}}{902+142.5 \mathrm{Bo}+\mathrm{Bo}^{2}} .
$$

The value of $S_{\text {cap }}$ versus Bo is compared to Princen's solution in Figure 7.

Equations (14) and (15) imply that the normalized thinning rate can be written as

$$
a=\frac{2 \pi}{9} \frac{(D / 2)^{2}}{S_{\text {cap }}}=\frac{1}{2^{2 / 39}} \frac{902+142.5 \mathrm{Bo}+\mathrm{Bo}^{2}}{94.7 \mathrm{Bo}+\mathrm{Bo}^{2}} .
$$

This "simple" model predicts the decrease of the normalized draining rate versus Bond number since the surface of the cap relative to the bubble size increases with Bond number (cf. Figure 7). The variation of $a$ versus Bo according to Eq. (16) is plotted in dashed line in Figure 5. In the limit of large Bo, this approximation agrees more quantitatively with experimental results than the analytical model of Debrégeas et al. ${ }^{12}$ presented in Sec. III B. Moreover, it allows to predict the evolution of thinning rate of the thin film in the limit of intermediate Bond number when the interface is not fully deformed by the bubble.

It is noteworthy that the limit of zero Bo corresponding to the case of a spherical bubble approaching a flat interface, the analytical solution of the Stokes flow allow to determine the lubrication force as a function of the film thickness, see for instance. ${ }^{32}$ According to the method proposed by Cox and Brenner ${ }^{33}$ detailed in the book of Kim and Karrila, ${ }^{34}$ Pigeonneau and Sellier ${ }^{17}$ point out that the lubrication force diverges following a logarithmic singularity leading to the collapse of the liquid film over a finite time. Thus, this asymptotic behavior is coherent with the divergence of (14) as well as the sharp increase of $a$ observed experimentally in the limit of Bo $\rightarrow 0$.

We note that the thinning rate expression (Eq. (16)) is based on the assumption of a uniform film thickness. Despite this first order approximation, it captures the effect of Bond number as it considers the variation of size of the cap on top of a bubble relative to its initial surface. The disagreement in the limit of large Bo between the numerical simulation and Eq. (16) is explained by the fact that the 
capillary suction from the curved interface at the bottom of the film (Plateau border) is neglected in the present model. Taking into account the capillary suction would increase the thinning rate.

\section{CONCLUSION}

Experiments of gas bubble pushed upward by gravity towards a free surface of a liquid with "bare" interface have been investigated. Four liquids are studied: the two firsts are synthetic and natural oils and the two lasts are molten glasses. Two specific experimental set-ups are developed working at room temperature for the investigations on oils and at high temperature for molten glasses. After the free rising of the observed bubble, the liquid film between the bubble and the free surface of the liquid is determined by an interferometry method.

For both kinds of liquids, we show that the liquid drains exponentially with time. This result is important for molten glass as it shows that over the time where the drainage occurs the molten glass interfaces are totally mobile. Moreover, the thinning rate corresponding to the logarithmic temporal derivative of the film thickness is determined. This quantity is normalized by a time scale based on the balance of gravity and viscosity forces at the bubble scale. It is clearly established that the thinning rate is a function of the bubble size or more precisely function of the Bond number. The thinning rate increases when the Bond number decreases in agreement with previous numerical simulation. ${ }^{17}$ In other words, small bubbles (or bubbles in high surface tension liquid) drain relatively faster than large bubble (or bubbles in low surface tension liquid).

It is noteworthy, that the relative surface of this cap compared to the surface of the bubble increases with Bond number. Thus, the pressure in the film squeezed by the buoyant force of the bubble decreases with Bond number. Assuming extensional squeezed flow of the liquid within the cap, we provide an expression of the film thinning rate as a function of Bond number that describes experimental results.

Finally, finding a "model liquid" for molten glass has big consequences. Indeed, the experiments on molten glass are always a big challenge since they require high temperature leading to high energy consuming and difficulties to analyze the data as well. Moreover, many artifacts due to high temperature can increase the uncertainties. So, in conclusion, both Castor oil or UCON $\mathrm{UM}^{\mathrm{TM}}$ oil can be used to study the film drainage in agreement with the physics of molten glass until any chemical effects play a role.

\section{ACKNOWLEDGMENTS}

We would like to thank M. Vignes-Adler for the helpful advices on the bubble experiment and discussion. We are grateful for the experimental work of S. Metallaoui during his Master Internship, who provides results of film thinning at ambient temperature. We are also indebted to P.-H. Guering and L. Canova working at Saint-Gobain Recherche (Aubervilliers, France) for the design of the optical set-up and G. Gauthier from FAST lab (Orsay, France) for viscosity measurements of oils.

\section{APPENDIX: ASYMPTOTIC EXPRESSION OF CAP AREA AT LOW AND HIGH BOND NUMBER}

At small Bond number, corresponding to a bubble smaller than the capillary length defined by ${ }^{35}$

$$
\kappa^{-1}=\sqrt{\frac{\sigma}{\rho g}}
$$

the interface above the bubble is slightly deformed and the bubble keeps the spherical shape. Howell ${ }^{20}$ studied the bubble shape for small Bond number. Remark that the radius of curvature, $R_{\text {cap }}$, is the twice of bubble radius when the Bond number goes to zero since the liquid film has two interfaces. Using the asymptotic solution given in Ref. 20, the area of the spherical cap normalized by the 
bubble radius squared is given by

$$
\frac{S_{\text {cap }}}{(D / 2)^{2}}=\frac{\pi}{3} \mathrm{Bo}+\mathcal{O}\left(\mathrm{Bo}^{2}\right)
$$

Conversely, at large Bond number, corresponding to bubble size larger than the capillary length, the free surface is strongly deformed and the shape of bubble as well. Thus, the bubble at the free surface tends to a hemispherical cap of radius $R_{c a p}=2^{1 / 3}(D / 2)$ leading to the following limit:

$$
\lim _{\operatorname{Bo} \rightarrow \infty} \frac{S_{\text {cap }}}{(D / 2)^{2}}=2^{5 / 3} \pi \approx 9.974
$$

${ }^{1}$ D. Weaire and S. Hutzler, The Physics of Foams (Clarendon, Oxford, 1999).

${ }^{2}$ I. Cantat, S. Cohen-Addad, F. Elias, F. Graner, R. Höhler, O. Pitois, F. Rouyer, and A. Saint-Jalmes, Les Mousses. Structure et Dynamique (Belin, Paris, 2010).

${ }^{3}$ J. Kappel, R. Conradt, and H. Scholze, "Foaming behaviour on glass melts," Glastech. Ber. 60, 189-201 (1987), http://cat.inist.fr/?aModele=afficheN\&cpsidt=7820577.

${ }^{4}$ J. van der Schaaf and R. G. C. Beerkens, "A model for foam formation, stability, and breakdown in glass-melting furnaces," J. Colloid Interface Sci. 295, 218-229 (2006).

${ }^{5}$ Both, glass and lava are silicates.

${ }^{6}$ A. A. Proussevitch, D. L. Sahagian, and V. A. Kutolin, "Stability of foams in silicate melts," J. Volcanol. Geotherm. Res. 59, 161-178 (1993).

${ }^{7}$ K. Mysels, K. Shinoda, and S. Frankel, Soap Film: Studies of Their Thinning (Pergamon, New York, 1959).

${ }^{8}$ L. W. Schwartz and R. V. Roy, "Modeling draining flow in mobile and immobile soap films," J. Colloid Interface Sci. 218, 309-323 (1999).

${ }^{9}$ S. Hartland, "The coalescence of a liquid drop at a liquid-liquid interface. Part I: Drop shape," Trans. Instn. Chem. Eng. 45, T97-T101 (1967).

${ }^{10}$ S. Hartland, "The coalescence of a liquid drop at a liquid-liquid interface. Part II: Film thickness," Trans. Instn. Chem. Eng. 45, T102-T108 (1967).

${ }^{11}$ S. Hartland, "The coalescence of a liquid drop at a liquid-liquid interface. Part III: Film rupture," Trans. Instn. Chem. Eng. 45, T109-T114 (1967).

${ }^{12}$ G. Debrégeas, P.-G. de Gennes, and F. Brochard-Wyart, "The life and death of "bare" viscous bubbles," Science 279, 1704-1707 (1998).

${ }^{13}$ J. Senée, B. Robillard, and M. Vignes-Adler, "Films and foams of Champagne wines," Food Hydrocolloids 13, 15-26 (1999).

${ }^{14}$ S. G. Yiantsios and R. H. Davis, "On the buoyancy-driven motion of a drop towars a rigid surface or a deformable interface," J. Fluid Mech. 217, 547-573 (1990).

${ }^{15}$ G. Oldenziel, R. Delfos, and J. Westerweel, "Measurements of liquid film thickness for a droplet at a two-fluid interface," Phys. Fluids 24, 022106 (2012).

${ }^{16}$ K. Kumar, A. D. Nikolov, and D. T. Wasan, "Effect of film curvature on drainage of thin liquid films," J. Colloid Interface Sci. 256, 194-200 (2002).

${ }^{17}$ F. Pigeonneau and A. Sellier, "Low-Reynolds-number gravity-driven migration and deformation of bubbles near a free surface," Phys. Fluids 23, 092102 (2011).

${ }^{18}$ D. Y. C. Chan, E. Klaseboer, and R. Manica, "Film drainage and coalescence between deformable drops and bubbles," Soft Matter 7, 2235-2264 (2011).

${ }^{19}$ G. I. Barenblatt, Scaling (Cambridge University Press, Cambridge, 2003).

${ }^{20}$ P. D. Howell, "The draining of a two-dimensional bubble," J. Eng. Math. 35, 251-272 (1999).

${ }^{21}$ C. J. S. Petrie, "Extensional viscosity: A critical discussion," J. Non-Newtonian Fluid Mech. 137, 15-23 (2006).

${ }^{22}$ P. D. Howell, "Models for thin viscous sheets," Eur. J. Appl. Math. 7, 321-346 (1996).

${ }^{23}$ C. Isenberg, The Science of Soap Films and Soap Bubbles (Dover, New York, 1992).

${ }^{24}$ T. Lakatos, L.-G. Johansson, and B. Simmingsköld, "Viscosity temperature relations in the glass system $\mathrm{SiO}_{2}-\mathrm{Al}_{2} \mathrm{O}_{3}$ $\mathrm{Na}_{2} \mathrm{O}-\mathrm{K}_{2} \mathrm{O}-\mathrm{CaO}-\mathrm{MgO}$ in the composition range of technical glasses," Glass Technol. 13, 88-94 (1972).

${ }^{25}$ A. Dietzel, "Zusammenänge zwischen Oberflächenspannung und Struktur von Glasschmelzen," Kolloid Z. 100, 368-380 (1942).

${ }^{26}$ K. C. Lyon, "Calculation of surface tension of glasses," J. Am. Ceram. Soc. 27(6), 186-189 (1944).

${ }^{27}$ C. Rubenstein, "Factors for the calculation of the surface tension of glasses at 1200 C," Glass Technol. 5, 36-40 (1964).

${ }^{28}$ A. Kucuk, A. G. Clare, and L. Jones, "An Estimation of the surface tension for silicate glass melts at $1400^{\circ} \mathrm{C}$ using statistical analysis," Glass Technol. 40(5), 149-153 (1999).

${ }^{29}$ H. Scholze, Glass. Nature, Structures and Properties (Springer-Verlag, Berlin, 1990).

${ }^{30}$ H. M. Princen, "Shape of a fluid drop at a liquid-liquid interface," J. Colloid Interface Sci. 18, 178-195 (1963).

${ }^{31}$ H. Kočárková, "Stabilité des mousses de verre: Expériences à l'échelle d'une bulle ou d'un film vertical," Ph.D. dissertation (Université Paris-Est, Marne la Vallée, 2011) (in English).

${ }^{32}$ E. Bart, "The slow unsteady settling of a fluid sphere toward a flat fluid interface," Chem. Eng. Sci. 23, 193-210 (1968).

${ }^{33}$ R. G. Cox and H. Brenner, "The slow motion of a sphere through a viscous fluid towards a plane surface - II Small gap widths, including inertial effects," Chem. Eng. Sci. 22, 1753-1777 (1967).

${ }^{34}$ S. Kim and S. J. Karrila, Microhydrodynamics. Principles and Selected Applications (Dover, New York, 2005).

${ }^{35}$ P.-G. de Gennes, F. Brochard-Wyart, and D. Quéré, Gouttes, Bulles, Perles et Ondes (Belin, Paris, 2005). 